\title{
Solitons in a Disordered Anisotropic Optical Medium ${ }^{1}$
}

\author{
M. Chertkov ${ }^{1}$, I. Gabitov ${ }^{1}$, I. Kolokolov ${ }^{2}$, and V. Lebedev ${ }^{3}$ * \\ ${ }^{1}$ Theoretical Division, LANL, Los Alamos, NM 87545, USA \\ ${ }^{2}$ Budker Institute of Nuclear Physics, Siberian Division, Russian Academy of Sciences, \\ pr. Akademika Lavrent'eva 11, Novosibirsk, 630090 Russia \\ ${ }^{3}$ Landau Institute for Theoretical Physics, Russian Academy of Sciences, ul. Kosygina 2, Moscow, 117334 Russia \\ *e-mail:lwlebede@yahoo.com \\ Received October 29, 2001
}

\begin{abstract}
The radiation-mediated interaction of solitons in a one-dimensional nonlinear medium (optical fiber) with birefringent disorder is shown to be independent of the separation between solitons. The effect produces a potentially dangerous contribution to the signal lost. (0) 2001 MAIK "Nauka/Interperiodica".
\end{abstract}

PACS numbers: 42.65.Tg; 42.81.Dp

The propagation of a pulse through an optical fiber with randomly varying anisotropy is usually addressed in the context of the Polarization Mode Dispersion (PMD). PMD is signal broadening caused by inhomogeneity of the medium birefringence. In the linear case, the study of PMD was pioneered by Poole [1], who showed that the pulse broadens as the two principal states of polarization split under the action of the random birefringence (see also [2]). Mollenauer et al. have numerically studied a nonlinear model of birefringent disorder in [3], where it was shown that a soliton, launched into the birefringent fiber, does not split, but it does undergo spreading [3] (see also [4]). In this letter, we develop an analytical approach and confirm that a single soliton does degrade due to disorder in the birefringence. The degradation is observable once the soliton traverses the distance $z_{\text {degr }} \sim D^{-1}$, where $D$ stands for the strength of the noise in the birefringence, measured in units of the soliton width and period $(D \ll 1$ is assumed, the typical case for telecommunication fibers).

The major finding of this letter is a new phenomenon which occurs on scales much shorter than $z_{\text {degr }}$. We report that the interaction between solitons induced by their combined radiation (generated by disorder) is an important factor affecting the soliton dynamics. Initially stationary solitons experience a relative acceleration, $\sim D$. The intersoliton separation changes on the order of the soliton width at $z_{\text {int }} \sim 1 / \sqrt{D} \ll z_{\text {degr. }}$. We use and generalize here an approach developed previously to describe solitons interacting in an isotropic medium with fluctuating dispersion [5]. The soliton interaction, in the case of [5], decays algebraically. By contrast, in the anisotropic case discussed in this letter, the interaction is separation-independent. The reason is that, in

\footnotetext{
${ }^{1}$ This work was submitted by the authors in English.
}

this case, a different type of wave scatters from the solitons. In the isotropic case, the scattering of the radiated waves, emitted by a soliton, by another soliton is not refracted. In the anisotropic case, radiation from one soliton pushes (literally) the other soliton, because the scattering potential is not transparent.

Let us briefly formulate the problem. The electric field $\mathbf{E}$, corresponding to a wave packet carrying frequency $\omega$, can be decomposed into complex components $\mathbf{E}=2 \operatorname{Re}\left[\mathbf{E}_{\omega} \exp \left(i k_{0} z-i \omega t\right)\right]$, where $z$ is the coordinate along the fiber. Concomitant averaging over fast oscillations and over the structure of fundamental mode (a monomode regime is assumed) constitutes the coarse-grained description for the signal envelope described by the two-component complex field $\Psi_{\alpha}$, $\overline{\mathbf{E}}_{\omega}=\Psi_{1}(z) \mathbf{e}_{1}+\Psi_{2}(z) \mathbf{e}_{2}$, where $\mathbf{e}_{1,2}$ are unit vectors orthogonal to each other and to the waveguide direction. The averaging results in the envelope equation $[6,7]$

$$
\begin{gathered}
i \partial_{z} \Psi_{\alpha}-\Delta_{\alpha \beta} \Psi_{\beta}-i m_{\alpha \beta} \partial_{t} \Psi_{\beta}+\partial_{t}^{2} \Psi_{\alpha} \\
+\frac{4}{3}\left(\left|\Psi_{1}\right|^{2}+\left|\Psi_{2}\right|^{2}\right) \Psi_{\alpha}+\frac{2}{3}\left(\Psi_{1}^{2}+\Psi_{2}^{2}\right) \Psi_{\alpha}^{*}=0 .
\end{gathered}
$$

Here, the wave packet is subjected to dispersion in retarded time $t$ and to the Kerr nonlinearity, which is described by the last two terms on the left-hand side of (1). The matrix $\hat{\Delta}$ describes the differences in the wavevectors. The matrix $\hat{m}$ describes the anisotropy in the group velocity for the two distinct states of polarization (of the respective linear problem). The isotropy is broken in Eq. (1), because the core of any fiber is elliptic rather than circular in cross section. It is assumed in Eq. (1) that the dispersion term and the nonlinear term are isotropic, since in real fibers anisotropy of dispersion and nonlinearity is usually less important than the 
effects of anisotropy described by the matrices $\hat{\Delta}$ and $\hat{m}$. The coefficients of nonlinearity and dispersion are rescaled to unity; i.e., $t$ and $z$ are already dimensionless in Eq. (1). If the matrices $\hat{\Delta}$ and $\hat{m}$ are zero, the full problem is isotropic and Eq. (1) supports the constant polarization solution, e.g., $\Psi_{2}=0$. Then, the equation for $\Psi_{1}$ is the scalar nonlinear Shrödinger (SNLS) equation. The self-conjugate matrix $\hat{\Delta}$ is traceless, since the trace can be excluded by a simple phase transformation. The (also self-conjugate) matrix $\hat{m}$ is traceless, as Eq. (1) is written in the reference frame moving with the mean group velocity. Both $\hat{\Delta}$ and $\hat{m}$ may contain regular and disordered parts. In a polarization-maintaining fiber, at least one of the regular parts is nonzero. If the phase change between the two polarizations caused by a regular part (say $\hat{\Delta}_{\text {reg }}$ ) becomes $\sim 1$ on a scale $z_{\text {reg }}$, an additional averaging over the distances larger than $z_{\text {reg }}$ reduces Eq. (1) to $[6,7]$

$$
\begin{gathered}
\left(i \partial_{z}+\partial_{t}^{2}+2\left|\Psi_{1}\right|^{2}+2 \varepsilon\left|\Psi_{2}\right|^{2}\right) \Psi_{1} \\
=\left(\Delta_{1 \beta}+i m_{1 \beta} \partial_{t}\right) \Psi_{\beta},
\end{gathered}
$$

and analogously for $\Psi_{2}$. The quantities $\hat{\Delta}$ and $\hat{m}$ left in Eq. (2) represent random contributions. Generically, eigenvectors of $\hat{\Delta}_{\text {reg }}$ correspond to elliptic polarizations, and the corresponding eigenvalues are complex. The quantity $\varepsilon$ in Eq. (2) measures the degree of ellipticity, $2 / 3 \leq \varepsilon \leq 2$. In the degenerate limit of linear polarization (the eigenvectors are real), $\varepsilon=2 / 3$. Subsequent analysis is devoted to models (1) and (2) with $\hat{\Delta}=0$ and random zero mean $\hat{m}$. The anisotropy matrix $\hat{m}$ can be written in terms of Pauli matrices as follows: $\hat{m}=$ $\sum_{k} h_{k}(z) \hat{\sigma}_{k}$, where $k=1,2,3$ and the real field $h_{k}$ is a function of $z$ only because the disorder is frozen in the fiber. The correlation scale of the random field $h_{j}(z)$ is short. (It is typically constrained by the process of fiber pulling from a silica preform, cabling, and spooling into a bobbin). Therefore, according to the central limit theorem, $h_{j}(z)$ on the larger scales can be treated as a Gaussian random process. The noise intensity is described by the matrix $\hat{D}, D_{i k}=\int d z\left\langle h_{i}(z) h_{k}\left(z^{\prime}\right)\right\rangle$. One assumes that the isotropy is restored on average, $D_{i k} \propto$ $\delta_{i k}$. Then, the statistics of $\hat{m}$ is characterized by

$$
\left\langle h_{i}\left(z_{1}\right) h_{k}\left(z_{2}\right)\right\rangle=D \delta_{i k} \delta\left(z_{1}-z_{2}\right)
$$

Similarly, one assumes that $\hat{\Delta}=\sum_{k} b_{k}(z) \hat{\sigma}_{k}$ and $\left\langle b_{i}\left(z_{1}\right) b_{k}\left(z_{2}\right)\right\rangle=D_{b} \delta_{i k} \delta\left(z_{1}-z_{2}\right)$.
We start with the single-soliton story. One looks for a solution to Eq. (1) or (2) in the form

$$
\Psi_{\alpha}=\delta_{1 \alpha} \exp (i z) \cosh ^{-1} t+v_{\alpha} .
$$

For $v_{1,2}=0$, Eq. (4) represents a single-soliton solution of the ideal, $\hat{m}=0$, problem. If the disorder is weak, one can substitute Eq. (4) into Eq. (1) or (2) and linearize with respect to $v_{1,2}$ to get

$$
\left(\partial_{z}-i \hat{L}_{1,2}\right)\left(\begin{array}{c}
v_{1,2} \\
v_{1,2}^{*}
\end{array}\right)=\left(\begin{array}{l}
R_{1,2} \\
R_{1,2}^{*}
\end{array}\right) \frac{\tanh t}{\cosh t}+\left(\begin{array}{c}
Q_{1,2} \\
Q_{1,2}^{*}
\end{array}\right) \frac{1}{\cosh t},
$$

where $R_{1}=h_{3}, R_{2}=h_{1}+i h_{2}, Q_{1}=i b_{3}, Q_{2}=i b_{1}-b_{2}$, and $\hat{L}_{1,2}$ are differential (second order in $t$ ) operators of the linear Schrödinger type with soliton-shaped $(\propto 1 / \cosh )$ potentials. It is convenient to expand $v_{1,2}$ in series in eigenfunctions of the operators $\hat{L}_{1,2}$. Spectra of the operators are separated into continuous and discrete parts, $v_{1,2}=v_{1,2}^{(0)}+\tilde{V}_{1,2}$. The four zero modes of $\hat{L}_{1}$ are related to variations of the soliton amplitude, position, phase, and phase velocity. There is also a localized eigenmode of $\hat{L}_{2}$ identified with variations of the soliton polarization. In the case of model (1), the polarization eigenmode becomes a zero mode of $\hat{L}_{2}$ corresponding to the free rotation of polarization axes and an additional zero mode appears that is related to ellipticity. Some localized modes are subjected to the linear, first-order, in disorder response. Thus, the position of the soliton $y$ varies randomly in $z:\left\langle y^{2}\right\rangle=D z$. Secondorder effects in radiation lead to variations of the soliton amplitude $\eta$. From the conservation law, which accounts for the balance of "energy" among the soliton and the continuous radiation $\left(v_{1,2}=0\right.$ at $z=0$ is assumed), one derives $\tilde{D} \int_{0}^{z} d z^{\prime} \eta^{3}\left(z^{\prime}\right)=1-\eta$, where the left- and right-hand sides represent, respectively, the radiative and the soliton contributions to the energy balance, and $\tilde{D} \sim D$. The solution to the integral equation, valid at any $z$, is

$$
\eta=(1+2 \tilde{D} z)^{-1 / 2}
$$

[Note that the single-soliton radiation in the degenerate case of Eq. (2) with $\varepsilon=1$ was studied in [8], where analogues of the aforementioned integral equation were derived. The equation was analyzed in [8] under the assumption that $z d \eta / d z \ll 1$, which led to the answer that the soliton amplitude degradation valid at $z D \ll 1$ only, where it coincides with Eq. (6)].

We now turn to the multisoliton case. Only scales shorter than $z_{\text {degr }}=1 / \mathrm{D}$ are discussed, so the random walk of $y$ and the degradation of the soliton amplitude can be neglected. The same argument applies to the polarization angle $\phi$ in the case of model (2). In the isotropic model case (1), the jitter of $\phi$ becomes important 


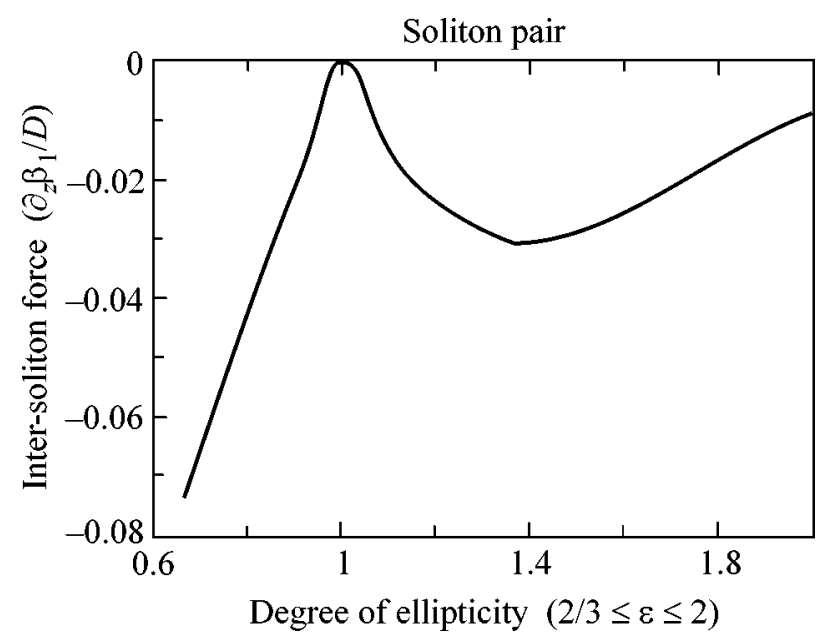

Fig. 1. Two solitons. Intersoliton force vs. degree of ellipticity.

at $z_{\phi} \sim 1 / D^{1 / 3}$. The effect, however, is collective: polarizations of different solitons rotate through the same angle, so that the relative polarization angle is unchanged at $z \ll z_{\text {degr }}$. We consider the $N$-soliton solution,

$$
\Psi_{\alpha}=\sum_{j=1}^{N} \exp \left[i \alpha_{j}+i \beta_{j}\left(t-y_{j}\right)\right] \cosh ^{-1}\left(t-y_{j}\right) \delta_{1 \alpha}+v_{\alpha}
$$

of Eqs. (1) and (2). One derives (and solves) the generalization of Eq. (5) and equations for the slow variables $y_{i}, \alpha_{i}$, and $\beta_{i}$, keeping in the latter the terms up to the second order in $v$. Direct averaging of the slow modes over the $h$-statistics is the next step. At $z \ll z_{\text {degr }}$, the relative phases $\alpha_{i}-\alpha_{j}$ do not change, while the soliton positions $y_{j}$ and phase velocities $\beta_{i}$ evolve according to

$$
\begin{gathered}
\partial_{z} y_{j}=-2 \beta_{j}, \quad \partial_{z} \beta_{j}=F_{j}, \\
F_{j}=\int d t U(t) \tanh \left(t-y_{j}\right) \cosh ^{-2}\left(t-y_{j}\right),
\end{gathered}
$$

where $U(t)$ is a quadratic form of $\tilde{V}, U(t)=4\left|\tilde{V}_{1}\right|^{2}+$ $\tilde{V}_{1}^{2}+\tilde{V}_{1}^{*^{2}}+2 \varepsilon\left|\tilde{V}_{2}\right|^{2}$ for model 2 . The force $F_{j}$ acting on the soliton is self-averaged at $z \gg 1$. Therefore, we come to a set of deterministic (like in classical mechanics) equations for the soliton positions and the phase velocities (the latter play the role of classical momenta). The general setting is familiar from [5]. However, the dependence of the intersoliton forces on the separation between the solitons in the polarization problems is different: the force does not depend on the separation. The key feature of the polarization problems is the refractive nature of $\hat{L}_{2}$, which is closely related to the nonintegrability of the no-disorder ( $\hat{m}=$ 0 ) problem in both of our settings (1), (2). This is in contrast to the integrability of SNLS, which is the no-

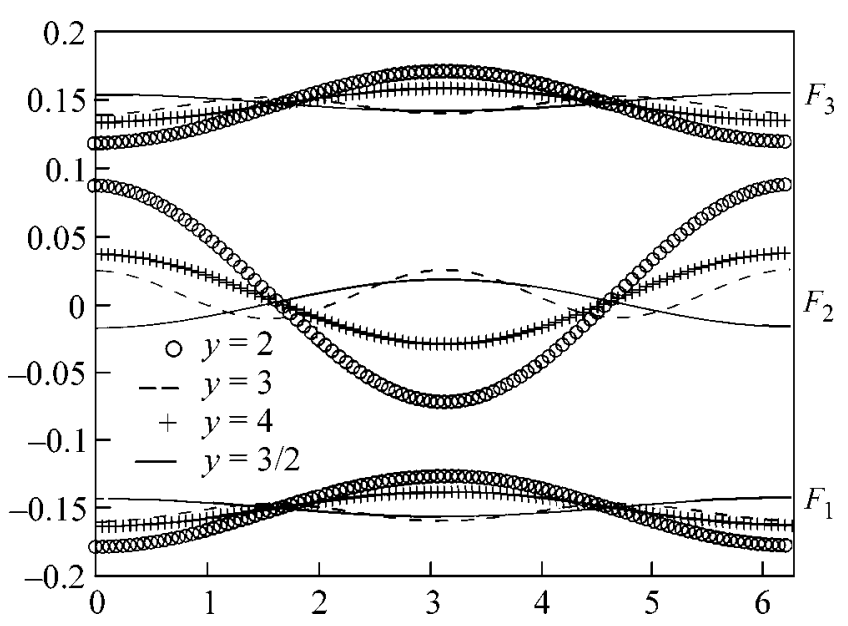

Fig. 2. Three solitons. Forces vs. intersoliton phase mismatch.

disorder limit of the scalar problem. Due to nonzero refraction, standing waves are formed in between the solitons in such a way that the wave amplitude does not depend on the intersoliton separation.

We present here quantitative results for model (2), obtained by numerical evaluation of the integral in Eq. (7) [with $U_{1,2}$ found via analytical integration of the generalized version of Eq. (5) and averaged over Eq. (3)]. A description of the calculation details will be published elsewhere. The $y$-independent $O(D)$ contribution to the intersoliton force for the two-soliton pattern is shown in Fig. 1. The force is independent of the phase mismatch, $\alpha_{1}-\alpha_{2}$. It is always negative (the solitons repel). The minimum value of the force is achieved at the boundary value, $\varepsilon=2 / 3$. The separation-independent contribution is zero at $\varepsilon=1$. This corresponds to transparent scattering, for the no-disorder limit is integrable in this case [9]. The independence of the force of the overall size of the soliton pattern persists in the multisoliton case, although a new feature-sensitivity to the phase mismatches-emerges. The dependence of the forces in the three-soliton pattern on the phase mismatch, in the special case $\alpha_{2}=0, \alpha_{3}=-\alpha_{1}=\alpha$, and $\epsilon=$ $2 / 3$, for various values of the relative separation, $y=$ $\left(y_{3}-y_{2}\right) /\left(y_{2}-y_{1}\right)$, is shown in Fig. 2. In the "symmetric" case, $y=1, F_{2}=0$, while $F_{3}=-F_{1}$, and the value is twice as large as the force acting on the second particle in the two-soliton case. In all other $y \neq 1$ situations, the forces do depend on $\alpha$. The values of the forces oscillate about the symmetric $(y=1)$ values.

To conclude, we have shown that the major destructive factor for a set of well-separated pulses in random birefrengent fibers is due to soliton-soliton interaction mediated by radiation. Note that the analytical method described in this paper can easily be generalized to a variety of more complicated sources of anisotropy in optical fibers. 
We are grateful to G.D. Doolen, E.A. Kuznetsov, and V.E. Zakharov for useful comments. This work was supported by the LDRD ER on "Statistical Physics of Fiber Optics Communications" at LANL, the J. R. Oppenheimer fellowship (MC), the DOE (contract W-7-405ENG-36) and its Program in AMS [KJ-01-01 (IG)], the Russian Foundation for Basic Research (project no. 00-02-17652), and the Russian Foundation for Promoting Science Personal Grant (IK).

\section{REFERENCES}

1. C. D. Poole, Opt. Lett. 13, 687 (1988); 14, 523 (1989); C. D. Poole, J. H. Winters, and J. A. Nagel, Opt. Lett. 16, 372 (1991).

2. N. Gisin and J. P. Pellaux, Opt. Commun. 89, 316 (1992).

3. L. F. Mollenauer et al., Opt. Lett. 14, 1219 (1989).
4. P. K. A. Wai, C. R. Menyuk, and H. H. Chen, Opt. Lett. 16, 1231 (1991); 16, 1735 (1991).

5. M. Chertkov, I. Gabitov, I. Kolokolov, and V. Lebedev, Pis'ma Zh. Ekssp. Teor. Fiz. 74, 391 (2001) [JETP Lett. 74, 357 (2001)].

6. A. L. Berkhoer and V. E. Zakharov, Zh. Éksp. Teor. Fiz. 58, 903 (1970) [Sov. Phys. JETP 31, 486 (1970)].

7. C. R. Menyuk, IEEE J. Quantum Electron. 25, 2674 (1989).

8. T. I. Lakoba and D. J. Kaup, Phys. Rev. E 56, 6147 (1997); M. Matsumoto, Y. Akagi, and A. Hasegawa, J. Lightwave Technol. 15, 584 (1997); L. F. Mollenauer et al., in Optical Fiber Telecommunications, Ed. by I. P. Kaminow and T. L. Koch (Academic, San Diego, 1997), Part IIIA; H. H. Chen and H. A. Haus, Opt. Lett. 25, 290 (2000).

9. S. V. Manakov, Zh. Éksp. Teor. Fiz. 65, 505 (1973) [Sov. Phys. JETP 38, 248 (1974)]. 